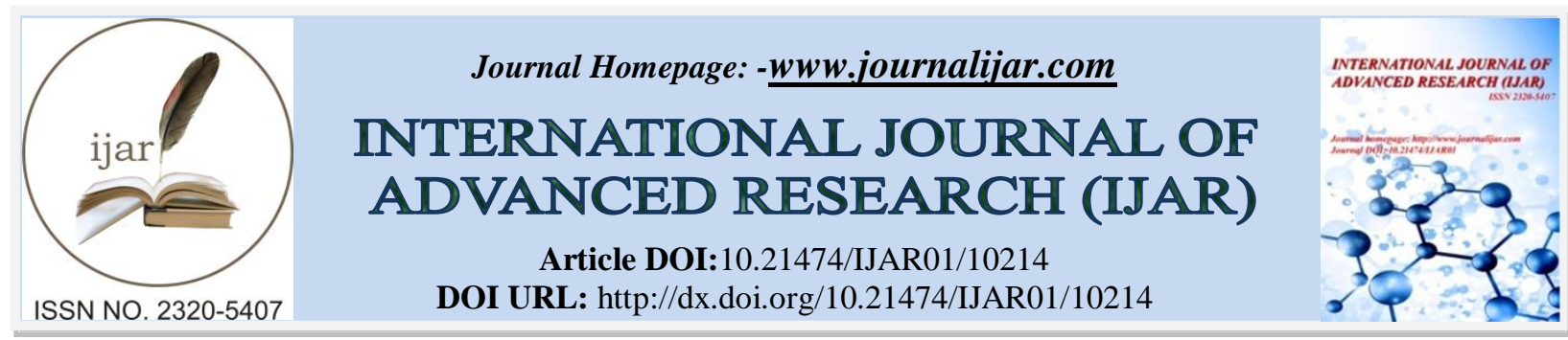

RESEARCH ARTICLE

\title{
INTERET DE LA PRISE EN CHARGE PRECOCE DU DERMATOFIBROSARCOME DE DARIER ET FERRAND (A PROPOS DE 30 CAS ET REVUE DE LA LITTERATURE).
}

\section{K. Ababou ${ }^{1}$, I. Moqfi ${ }^{1}$, F. Fouadi ${ }^{1}$, A. Khales ${ }^{1}$, Y. Ribag ${ }^{1}$, T. Nassim Sabah ${ }^{2}$, I. Moqfi ${ }^{1}$, MK. EL Khatib ${ }^{3}$ and S.} Siah $^{1}$

1. Service de Chirurgie Plastique, Réparatrice et des Brûlés, Hôpital Militaire d'Instruction Mohamed V, Rabat, Maroc.

2. Service de Chirurgie Plastique, Hôpital Militaire, Agadir, Maroc.

3. Service de chirurgie maxillo-faciale et stomatologie, Hôpital Militaire d'Instruction Mohamed V, Rabat, Maroc.

\section{Manuscript Info}

Manuscript History

Received: 10 October 2019

Final Accepted: 12 November 2019

Published: December 2019

\section{Abstract}

Introduction: Les dermatofibrosarcomes de Darrier et Ferrand sont des tumeurs rares, de malignité intermédiaire rarement métastatique et dont l'extension tumorale est souvent sous-estimée.

Matériel et Méthodes: une étude analytique de 30 patients qui ont été pris en charge dans notre formation pendant une durée de six ans et d'une revue de la littérature.

Résultats: patients jeunes, prédominance masculine, Tous nos patients ont bénéficié d'une exérèse chirurgicale large, avec des marges de sécurité classiques : $5 \mathrm{~cm}$ chez la majorité des patients.

Discussion: Nous mettons le point sur l'aspect épidémiologique de la maladie et les différentes modalités de prise en charge chirurgicale.

Objectif du travail: l'intérêt essentiel du geste initial ; une exérèse complète et précoce, une couverture simple par une greffe cutanée ; permettent un suivi régulier des patients en post-opératoire.

Copy Right, IJAR, 2019,. All rights reserved.

\section{Introduction:-}

Le dermatofibrosarcome de Darrier et Ferrand (DFS) bien que rare est le sarcome cutané le plus fréquent. Il s'agit d'une tumeur lentement évolutive, classée comme une tumeur de malignité intermédiaire rarement métastatique [1], donc d'agressivité essentiellement locale, mais son invasion infraclinique souvent importante est responsable de récidives fréquentes. La forme protuberans de Darier-Ferrand (DFSP) est la plus connue, mais il existe des formes non protuberans de diagnostic souvent tardif. L'évolution peut être marquée par une transformation avec apparition de foyer(s) de fibrosarcome. Le DFS atteint principalement des sujets jeunes. Sa localisation préférentielle est le tronc et les membres.Nous rapportons une série de 30 patients, opérés au service sur une période de six ans.

\section{Matériel et Méthodes:-}

À partir d'une étude rétrospective étalée sur six ans ; depuis janvier 2013 au 31 décembre 2018, nous avons étudié 30 patients présentant des Dermatofibrosarcomes histologiquement prouvés ; et qui ont étaient opérés service de chirurgie plastique de l'hôpital Militaire Mohamed V de Rabat .

Corresponding Author:- K. Ababou

Address:- Service de Chirurgie Plastique, Réparatrice et des Brûlés, Hôpital Militaire d'Instruction Mohamed V, Hay riad 10100, Rabat, Maroc 
Les critères d'inclusions : Tous les patients documentés et inscrits dans notre registre ;présentant un dermatofibrosarcome de localisations variés : et qui ont bénéficiés de prise en charge chirurgicale dans notre formation durant la période pré-citée.

Les critères d'exclusion : les patients présentant des dermatofibrosrcomes, opérés et perdus de vue en postopératoire.

Les données analysées ont été l'âge, le sexe, La présence d'antécédents médicaux (Diabète et tares) et chirurgicaux , le siège et la taille de la tumeur, l'extension profonde, les techniques chirurgicales utilisés, le suivi et l'évolution à court terme, à moyen et à long terme ; pour guetter des complications éventuelles et des récidives locales.

Les résultats fonctionnels et esthétiques ont été jugés par le chirurgien et les patients en évaluant leurs satisfactions sur le plan fonctionnel et esthétique en se basant sur une fiche d'évaluation.

\section{Résultats:-}

L'âge moyen était de 42,2 ans allant de 11 à 64 ans, le sexe ratio était de 0,63 avec une prédominance masculine. Le dos était la localisation préférentielle avec $33,33 \%$ des cas, suivi de la face antérieure du thorax avec $23,33 \%$ des cas, l'abdomen avec $13,33 \%$ des cas,

Les membres supérieurs et le cuir chevelu avec $10 \%$ des cas et en dernier les membres inférieurs avec 6,66\% des cas.Tous nos patients ont bénéficié d'une exérèse chirurgicale large, avec des marges de sécurité :

Latérales de $5 \mathrm{~cm}$ chez 28 patients, de $3 \mathrm{~cm}$ chez 2 patients porteurs d'un DFS au niveau de l'extrémité céphalique. Et en profondeur emportant la première barrière anatomique saine.

Les techniques de Couverture de la perte de substance engendrée par l'exérèse chirurgicale étaient les suivantes : Greffes deromo-epidermiques semi épaisses dans 28 cas, et suture directe dans 2 cas.Une récidive locale était notée chez un seul patient. Le recul moyen est de 5,3 ans avec des extrêmes allant de 1 à 11 ans. Sur les 30 patients opérés dans notre service, aucune métastase n'a été décelée.

\section{Discussion:-}

L'incidence estimée du DFS est de l'ordre de 0,8 à 4,2 cas/millions d'habitants par an [2].Le diagnostic est habituellement porté entre 30 et 50 ans (toutefois les âges extrêmes se situent de l'adolescence jusqu'à 90 ans et plus). Les formes pédiatriques sont rares (moins de $6 \%$ ) et doivent absolument être confirmées par un pathologiste expérimenté. Il existe une très légère prédominance masculine [3 4] .

Le risque de rechute métastatique est faible de l'ordre de $5 \%$ [5 6] . Globalement le pronostic carcinologique est bon avec une survie globale de $92 \%$ à 5 ans. Cela dit ce pronostic est directement lié à la qualité de la prise en charge [7] .

Historiquement, compte tenu du caractère invasif et spéculé de la tumeur, la chirurgie était basée sur une exérèse large, jusqu'à $5 \mathrm{~cm}$ en périphérie de la tumeur, mais cependant sans spécificité sur les marges idéales en profondeur. Ce sacrifice était le seul capable d'assurer un risque faible de récidive locale, faisant office donc de standard. Quelques séries comportant des patients avec des marges supérieures à $5 \mathrm{~cm}$ font état d'un risque de rechute quasi nul [8 9] , sans précision sur les éventuelles séquelles associées. À l'opposé, en cas de résection marginale (R1), le taux de récidive locale peut grimper à plus de $60 \%$ [10] . D'autres séries publiées avec des marges d'exérèse allant de 2 à $5 \mathrm{~cm}$ retrouvent un risque de rechute locale de l'ordre de $6 \%$ [6 11112 .

Dès lors, les chirurgiens cherchent à optimiser la marge idéale de résection. Ainsi, la technique de Mohs centrée sur l'analyse extemporanée des marges s'est rapidement développée. La technique initiale a été décrite en 1941 ( zinc chloride chemosurgery ), secondairement améliorée en 1974 par l'analyse extemporanée des marges de résection [13] . Cette technique chirurgicale avec analyse immédiate des berges saines permet d'améliorer le résultat de l'analyse histologique en la standardisant mais aussi de limiter le sacrifice chirurgical [ 141516 ] . Cependant, cette chirurgie " micrographique », sur prélèvement congelé, imposait soit une expertise histologique par le chirurgien, soit une étroite collaboration entre chirurgien et anatomo-pathologiste, avec une analyse fastidieuse en temps réel de 
l'intégralité du prélèvement, curetage de la tumeur clinique ( debulking ), excision en forme de « saladier » et orientation topographique des berges puis coupes au cryostat avec réalisation de coupes parallèles à l'épiderme depuis la face hypodermique jusqu'à l'épiderme afin de réaliser une analyse tri- dimensionnelle des berges. Ces contraintes ont amené, dès 1988, à modifier la technique et à plutôt réaliser une analyse secondaire sur paraffine, plus lente mais plus précise (appelée le «slow-Mohs » ou technique de Breuninger [17] ). Cette analyse «3Dhistology » avait l'avantage d'optimiser le temps opératoire en limitant l'attente des résultats, mais aussi de respecter les protocoles standards anatomopathologiques. Deux variantes techniques ont été décrites pour le slow-Mohs : « en muffin », où l'emballage du muffin est décollé au pourtour puis analysé, ou " en gâteau », où c'est le moule du gâteau qui est séparé pour étudier les berges.

Les résultats oncologiques après congélation (Mohs) ou fixation sur paraffine ( slow-Mohs ) ont été comparés rétrospectivement par Breuninger, ne retrouvant aucun avantage à la technique initiale de Mohs, faisant conclure en faveur de la technique standard sur paraffine, bien plus facile à réaliser [18] Farma et al. ont rapporté leur expérience de chirurgie micrographique avec des marges allant de 1 à $2 \mathrm{~cm}$ pour 2014 patients et soulignent l'importance de l'examen méticuleux des marges en anatomopathologie. Afin d'obtenir des marges négatives, certains patients ont été parfois opérés jusqu'à 4 fois (médiane d'une intervention néanmoins). Une radiothérapie adjuvante a même été proposée pour 9 patients (dans 6 cas pour une exérèse marginale sans reprise chirurgicale possible). Le taux de récidive locale était de $1 \%$ avec un suivi médian de 64 mois [19].

Cette tumeur est considérée comme chimio-résistante bien que des réponses anecdotiques ont été rapportées avec le méthotrexate [20]. Inhibant le PDGFbR (mais aussi Kit, Abelson et CSF1), l'imatinib était à évaluer dans le traitement du dermatofibrosarcome. Les premiers cas rapportés dans la littérature ont montré que seules les tumeurs présentant le transcrit de fusion répondent à l'imatinib.

Il n'y a pas d'essai randomisé comparant chirurgie micrographique de Mohs (ou slow-Mohs ) versus chirurgie large avec analyse méticuleuse des marges. Une méta-analyse a été réalisée par Foroozan et al. ; 19 études (essentiellement rétrospectives) non randomisées ont été analysées. Le suivi médian était de 68 mois avec des taux de rechute locale de 1,1\% avec la chirurgie micrographique de Mohs (IC95\%:0,0-6,0\%) contre 6,3\% avec la chirurgie large (IC95\%:3,2-11,0\%) [21].

\section{Dans notre série:}

la chirurgie était basée sur une exérèse large avec des marges latérales de $5 \mathrm{~cm}$ pour $28 \mathrm{cas}$ et $3 \mathrm{~cm}$ pour 2 cas (tumeur localisée au niveau de l'extrémité céphalique) emportant en profondeur une barrière anatomique saine.

\section{Sur 18 patients vus en première intention, aucun cas de récidive:}

Sur les 12 patients traités en deuxième intention, on note un seul cas de récidive, il s'agit de la patiente porteuse d'un dermatofibrosarcome du cuir chevelu récidivant.

\section{Cette constatation témoigne de l'importance du premier acte chirurgical:}

la reconstruction par lambeaux ne peut être envisagée que lorsque nous disposons d'un diagnostic histologique de certitude.

Cependant la greffe de peau semi-épaisse a été réalisé chez 28 patients et la suture directe chez 2 patients seulement. Ces derniers présentaient des dermatofirosarcomes de la paroi abdominale.

\section{Dans notre série, la totalité des patients ont bénéficié d'un suivi strict:}

C'est la raison pour laquelle il conviendrait de bien former les médecins traitants, au cours des diplômes universitaires et des congrès sur la prise en charge ce type de pathologie et contribuer à une information large de la population ;permettant ainsi un diagnostic précoce. 


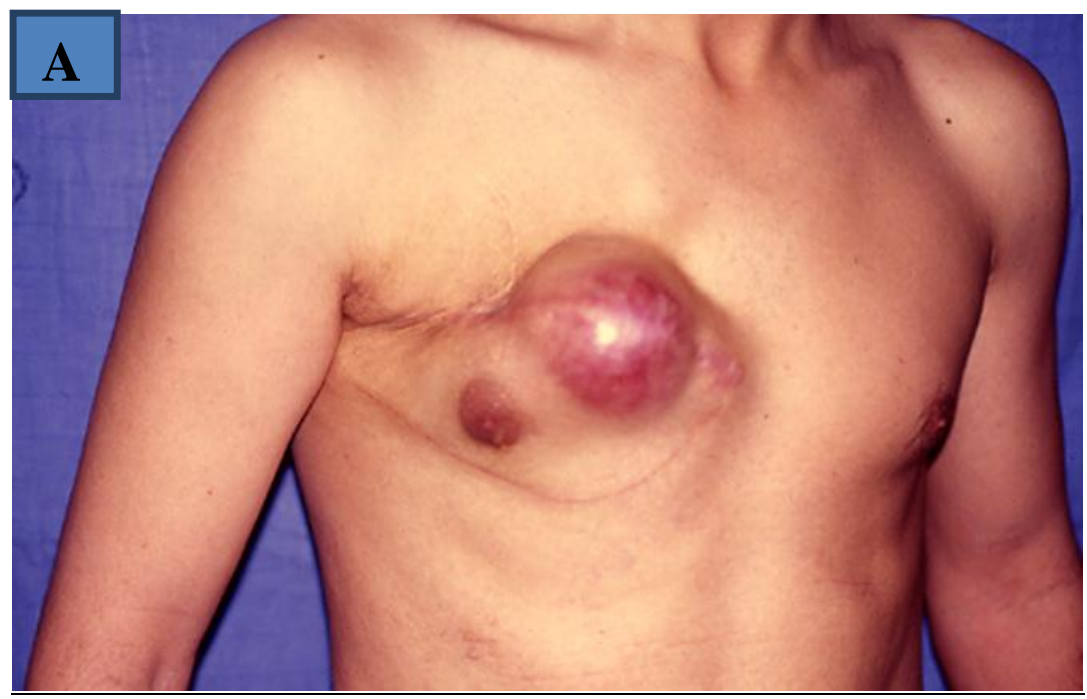

Figure 1:- Aspect initial de la tumeur.

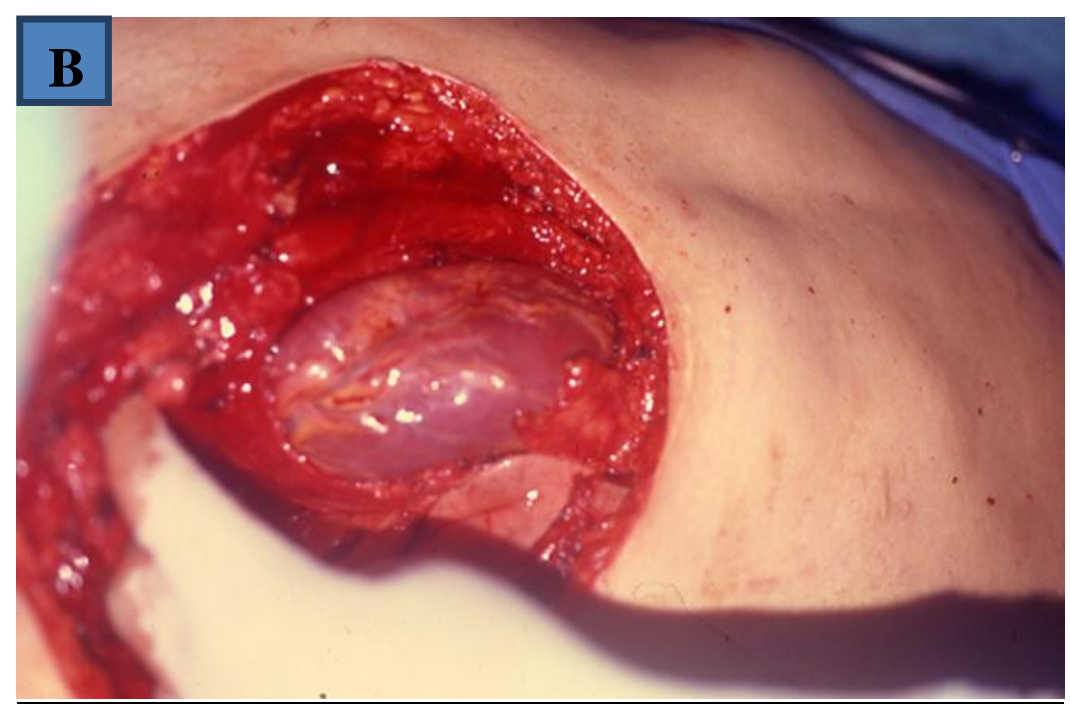

Figure 2:- Aspect après exérèse tumorale large.

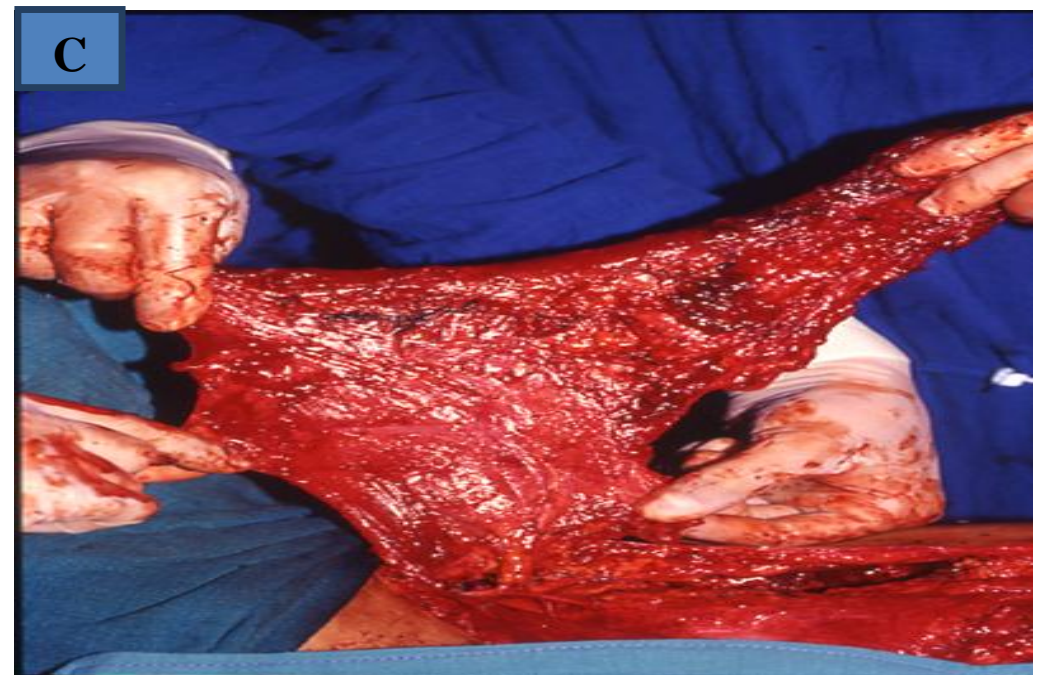

Figure 3:- DFS du tronc ,mobilisation d'un lambeau grand dorsal pédiculé pour couverture de la perte de substance. 


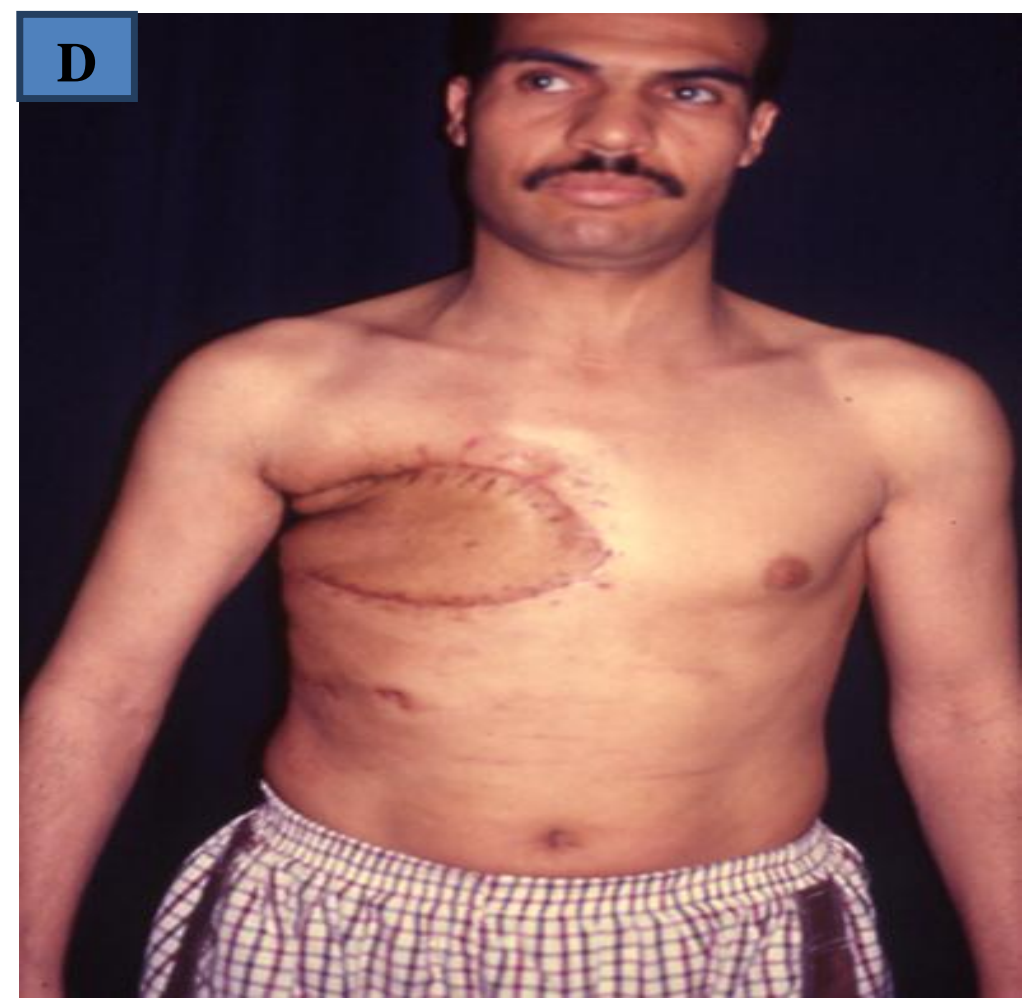

Figure 4:- DFS du tronc, Aspect post opératoire après 3mois.

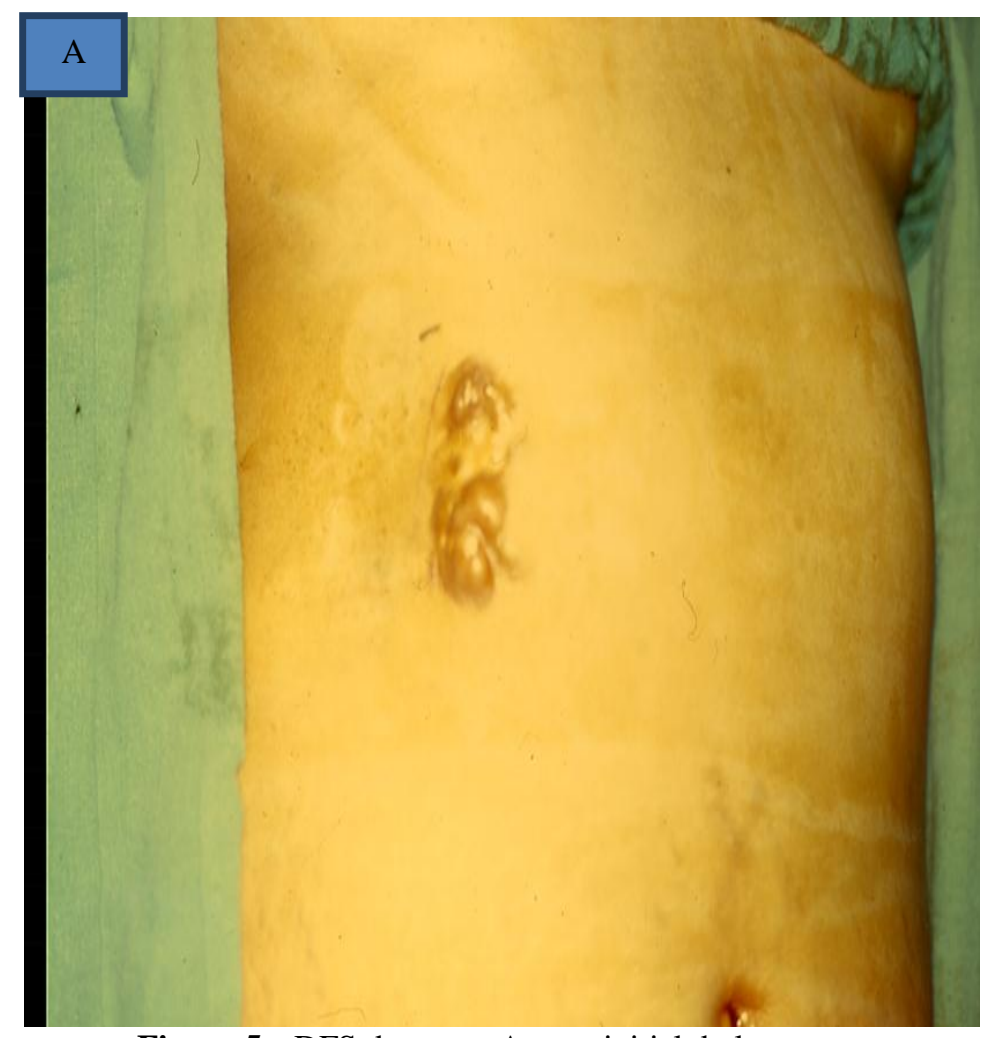

Figure 5:- DFS du tronc, Aspect initial de latumeur. 


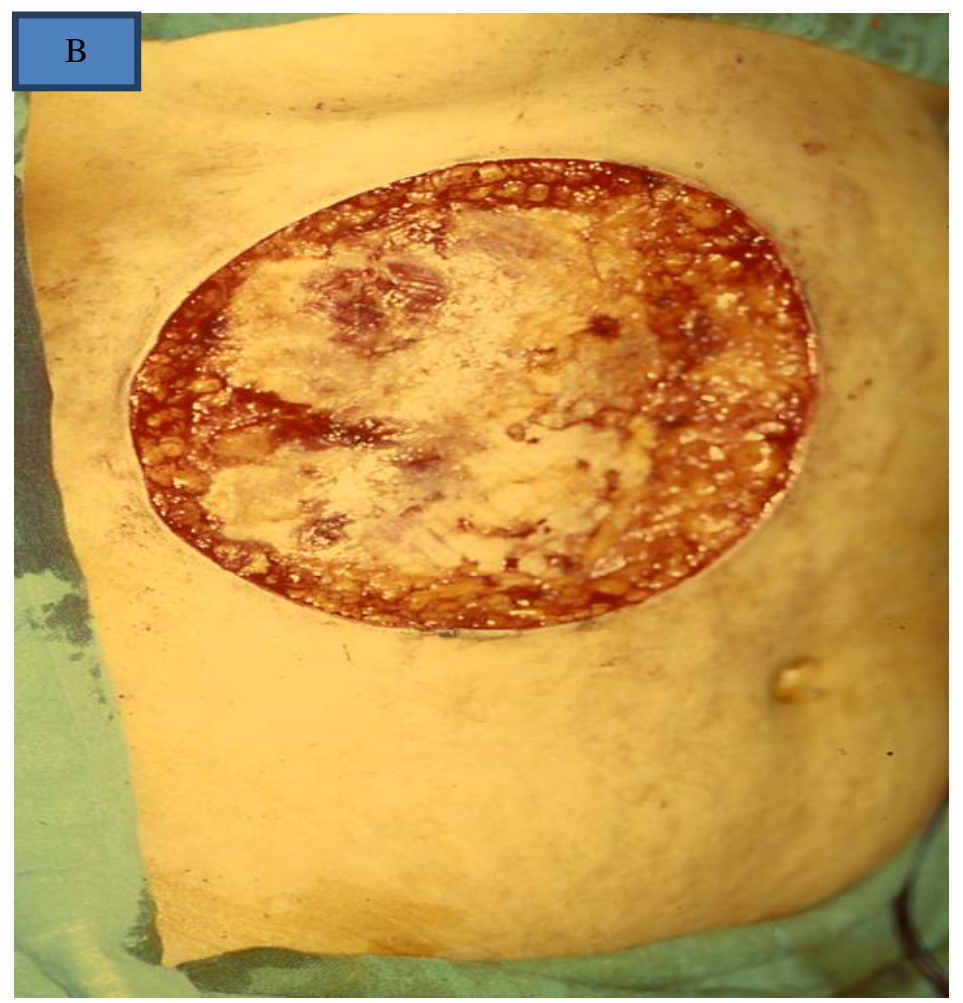

Figure 6:- DFS du tronc, Aspect après exérèse tumorale large.

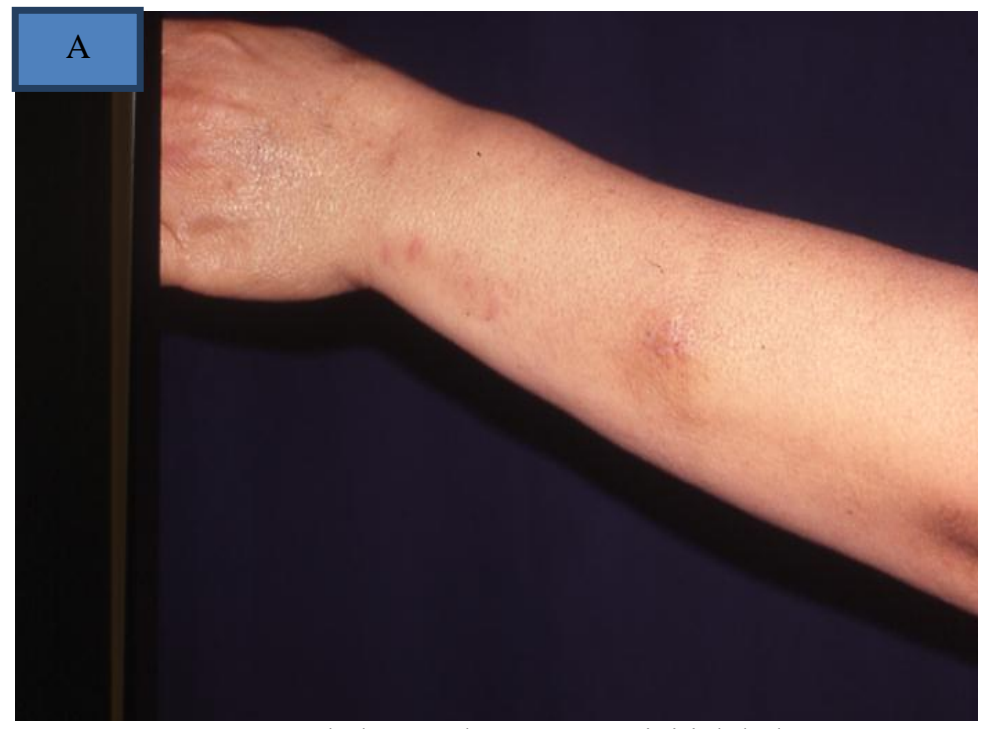

Figure A:- DFS de l'avant bras, Aspect initial de la tumeur. 


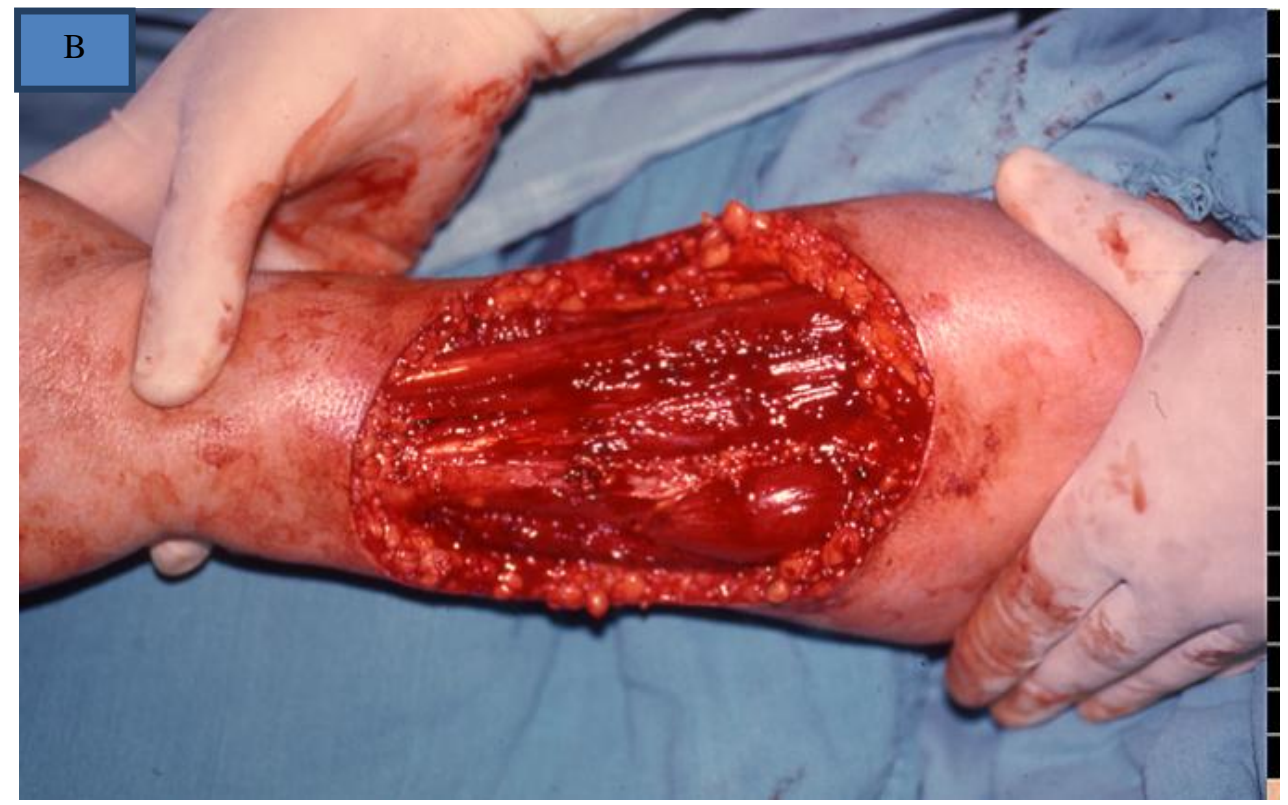

Figure B:- Aspect après exérèse tumorale large.

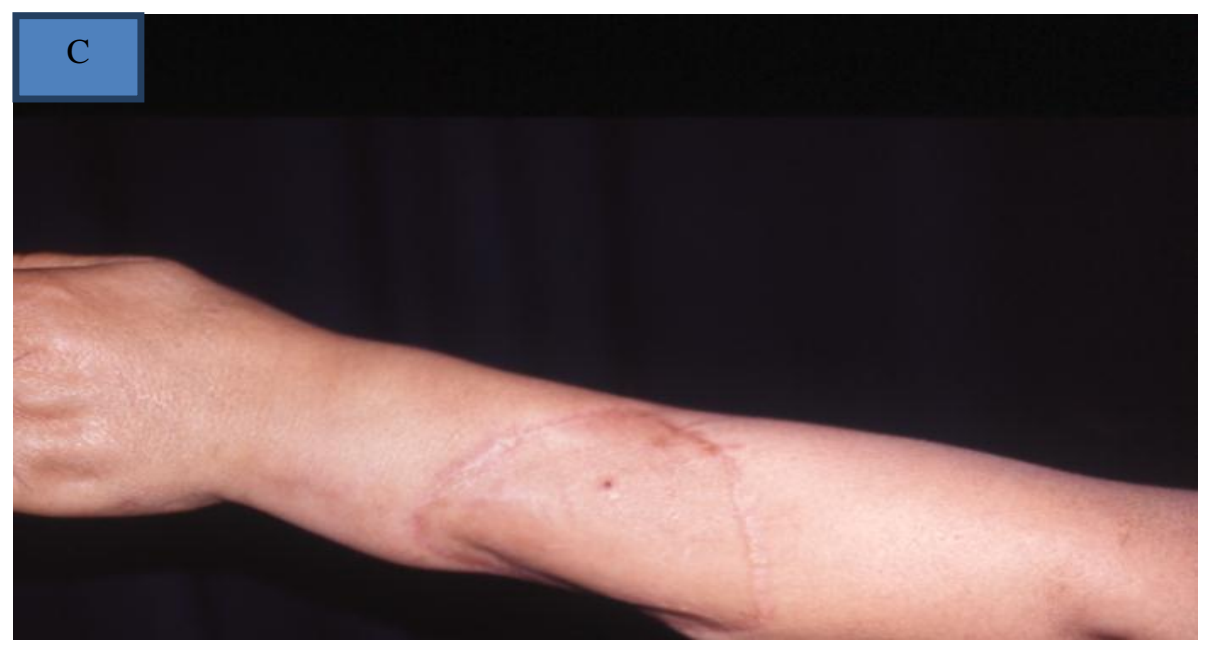

Figure C:- Aspect à 6 mois de la couverture de la perte de substance par une greffe de peau demi-epaisse.

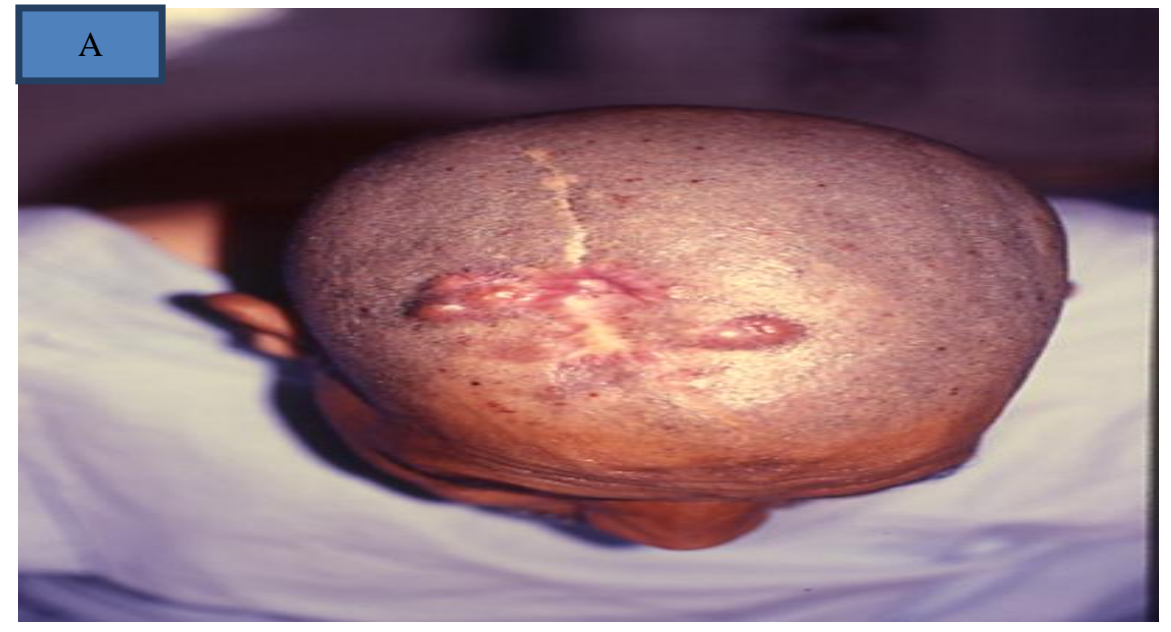


Figure A:- DFS du cuir chevelu, Aspect initial de la tumeur.

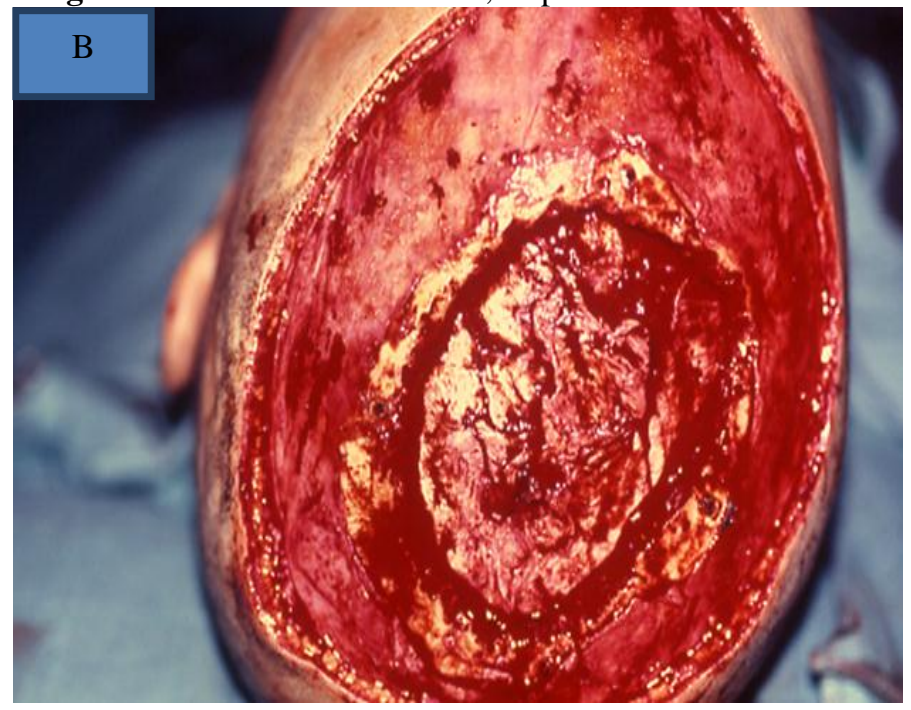

Figure B:- Aspect après exérèse tumorale large.

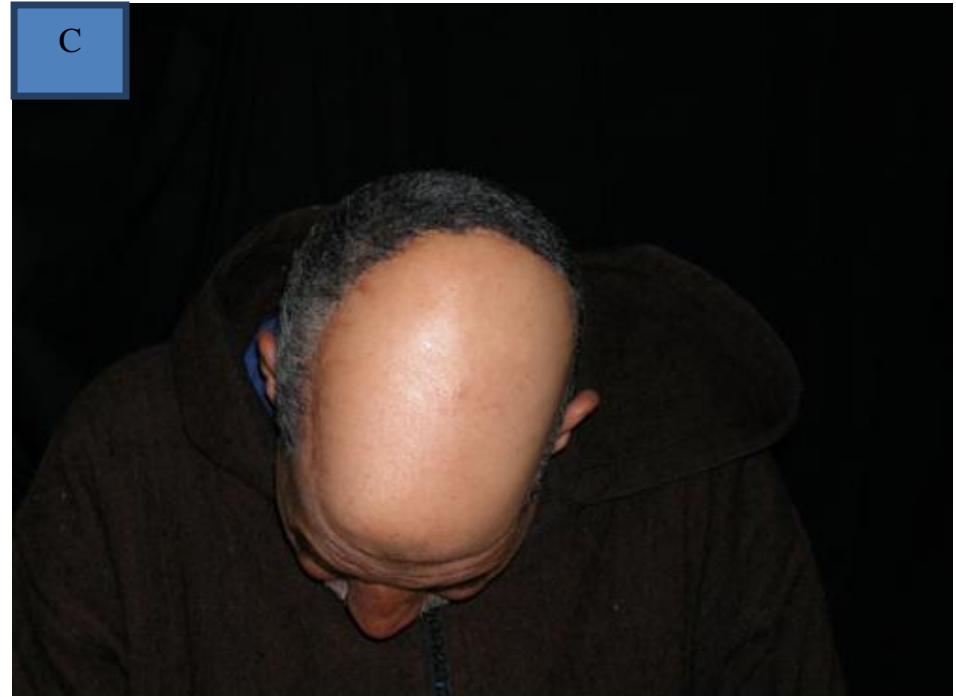

Figure C:- Aspect après 3mois avec couverture de la perte de substance par un lambeau de grandd dorsal libre.

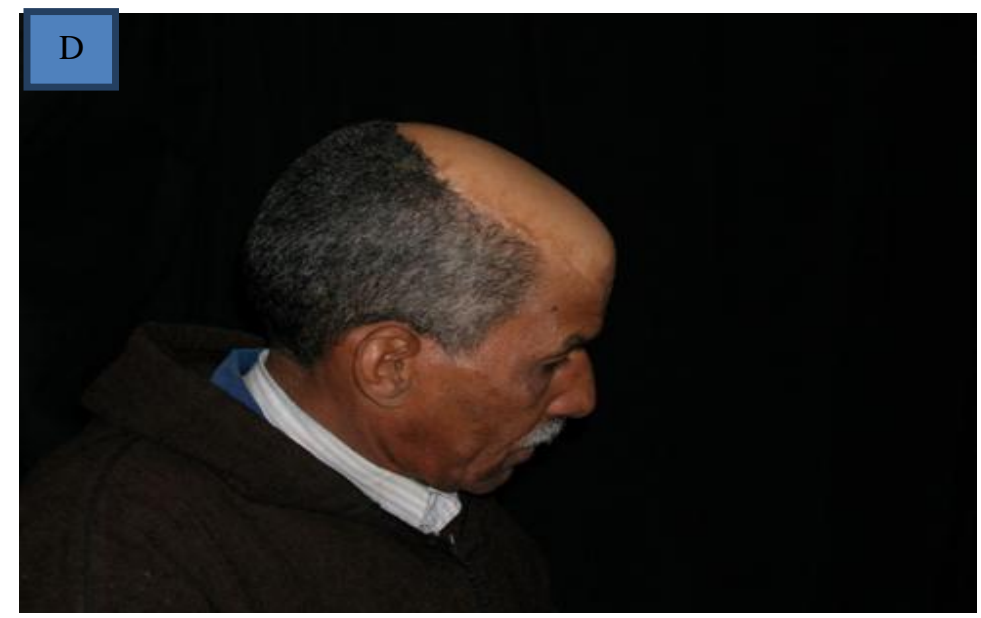

Figure D:- Aspect après 3mois avec couverture de la perte de substance par un lambeau de grandd dorsal libre. 


\section{Conclusion:-}

Le Dermatofibrosarcome de Darier et Ferrand est une tumeur cutanée rare, à évolution locale lente. Le risque évolutif de cette maladie est probablement plus lié aux récidives locales qu'aux métastases qui sont exceptionnelles.

Le diagnostic, souvent évoqué lors de l'examen clinique, repose sur l'étude histologique et peut, en cas de doute, être confirmé par une étude immunohistochimique.

Le traitement du DFS est exclusivement chirurgical et consiste en une exérèse large, mais exige néanmoins une surveillance clinique à vie sachant que certains cas de récidives ont été décrits tardivement.

\section{Références:-}

1. Fletcher C.D.M., Bridge J.A., Hogendoorn P., and Mertens F.: In Elder D.E., Massi D., Scolyer R.A., and Willemze R. (eds): WHO classification of tumors of soft tissue and bone.

2. Criscione V.D., and Weinstock M.A.: Descriptive epidemiology of dermatofibrosarcoma protuberans in the United States, 1973 to 2002. J Am Acad Dermatol 2007; 56: pp. 968-973

3. Bowne W.B., Antonescu C.R., Leung D.H., Katz S.C., Hawkins W.G., Woodruff J.M., et al: Dermatofibrosarcoma protuberans: a clinicopathologic analysis of patients treated and followed at a single institution. Cancer 2000; 88: pp. 2711-2720

4. Rutgers E.J., Kroon B.B., Albus Lutter C.E., and Gortzak E.: Dermatofibrosarcoma protuberans: treatment and prognosis. Eur J Surg Oncol 1992; 18: pp. 241-248

5. Chang C.K., Jacobs I.A., and Salti G.I.: Outcomes of surgery for dermatofibrosarcoma protuberans. Eur J Surg Oncol 2004; 30: pp. 341-345

6. Fiore M., Miceli R., Mussi C., Lo Vullo S., Mariani L., Lozza L., et al: Dermatofibrosarcoma protuberans treated at a single institution: a surgical disease with a high cure rate. J Clin Oncol 2005; 23: pp. 7669-7675

7. Corey R.M., Swett K., and Ward W.G.: Epidemiology and survivorship of soft tissue sarcomas in adults: a national cancer database report. Cancer Med 2014; 3: pp. 1404-1415

8. Arnaud E.J., Perrault M., Revol M., Servant J.M., and Banzet P.: Surgical treatment of dermatofibrosarcoma protuberans. Plast Reconstr Surg 1997; 100: pp. 884-895

9. Lemm D., Mugge L.O., Mentzel T., and Hoffken K.: Current treatment options in dermatofibrosarcoma protuberans. J Cancer Res Clin Oncol 2009; 135: pp. 653-665

10. Rutgers E.J., Kroon B.B., Albus-Lutter C.E., et al: Dermatofibrosarcoma protuberans: treatment and prognosis. Eur J Surg Oncol 1992; 18: pp. 241-248

11. DuBay D., Cimmino V., Lowe L., Johnson T., and Sondak V.: Low recurrence rate after surgery for dermatofibrosarcoma protuberans: a multidisciplinary approach from a single institution. Cancer 2004; 100: pp. 1008-1016

12. Paradisi A., Abeni D., Rusciani A., Cigna E., Wolter M., Scuderi N., et al: Dermatofibrosarcoma protuberans: wide local excision vs. Mohs micrographic surgery. Cancer Treat Rev 2008; 34: pp. 728-736

13. Tromovitch T.A., and Stegman S.J.: Microscopie-controlled excision of cutaneous tumors: chemosurgery, fresh tissue technique. Cancer 1978; 41: pp. 653-658

14. Wacker J., Khan Durani B., and Hartschuh W.: Modified Mohs micrographic surgery in the therapy of dermatofibrosarcoma protuberans: analysis of 22 patients. Ann Surg Oncol 2004; 11: pp. 438-444

15. Meguerditchian A.N., Wang J., Lema B., Kraybill W., Zeitouni N., and Kane J.: Wide excision or Mohs micrographic surgery for the treatment of primary dermatofibrosarcoma protuberans. Am J Clin Oncol 2010; 33: pp. 300-303

16. Serra-Guillen C., Llombart B., Nagore E., Guillen C., Requena C., Traves V., et al: Mohs micrographic surgery in dermatofibrosarcoma protuberans allows tumour clearance with smaller margins and greater preservation of healthy tissue compared with conventional surgery: a study of 74 primary cases. Br J Dermatol 2015; 172: pp. 1303-1307

17. Boehringer A., Adam P., Schnabl S., Häfner H.M., and Breuninger H.: Analysis of incomplete excisions of basal-cell carcinomas after breadloaf microscopy compared with 3D-microscopy: a prospective randomized and blinded study. J Cutan Pathol 2015; 42: pp. 542-553

18. Breuninger H.: Micrographic surgery of malignant skin tumors: a comparison of the frozen technique with paraffin sectioning. Facial Plast Surg 1997; 13: pp. 79-82

19. Farma J.M., Ammori J.B., Zager J.S., Marzban S.S., Bui M.M., Bichakjian C.K., et al: Dermatofibrosarcoma protuberans: how wide should we resect? Ann Surg Oncol 2010; 17: pp. 2112-2118

20. Mendoza CB, Gerwig WH, Watne AL. Dermatofibrosarcoma protuberans with metastases treated with methotrexate. Am J Surg 1970;120:119-21.

21. Foroozan M., Sei J.F., Amini M., Beauchet A., and Saiag P.: Efficacy of Mohs micrographic surgery for the treatment of dermatofibrosarcoma protuberns: systematic review. Arch Deramtol 2012; 148: pp. 1055-1063. 